\title{
Editorial
}

\section{What is new in ESC 2021 guidelines on prevention of cardiovascular diseases?}

The updated ESC guidelines for the prevention of cardiovascular diseases (CVD) were published in August 2021.

The most significant news's are:

- The new guidelines give more attention to CVD prevention in older persons (70 years and older). For them, it was suggested to use a separate scale SCORE2-OP.

- The guidelines introduce a new, step-wise approach to intensify treatment based on cardiovascular risk, the benefits of treatment against risk factors, risk modifiers, comorbidities and patient preferences.

- The guidelines also provide age-stratified risk thresholds for otherwise healthy individuals and assess the risk of cardiovascular disease and the benefits of treatment. So, depending on age, a low-to-moderate 10 -year risk of complications is $<2.5 \%$ (people under 50 ); $<5 \%$ (people $50-69$ years old) or $<7.5 \%$ (over 70 years old).

- Also, a guideline states that bariatric surgery should be considered for obese people with an increased risk of cardiovascular disease when healthy diet and exercise do not result in continued weight loss (Ila, B).
- For patients with atherosclerotic cardiovascular disease (ASCVD), low doses of colchicine ( $0.5 \mathrm{mg}$ daily) may be considered for secondary prevention, especially if other risk factors are not well controlled (IIb, A). In patients with a high or very high risk of complications and triglyceride levels $>1.5 \mathrm{mmol} / \mathrm{L}$, despite statin treatment and lifestyle changes, icosapent ethyl 2-4 g daily (in combination with statins) may be considered (IIb, B).

- 2016 Recommendations indicated the negative impact of air pollution on the forecast of CVD. The updated guidelines are more specific about the effects of air pollution: patients at very high risk of CVD are advised to avoid prolonged contact with areas with high levels of air pollution (IIb, C).

- In patients with diabetes mellitus in combination with ASCVD, it is recommended to prescribe glucagon like peptide-1 receptor agonists (GLP-1RA) or sodium glucose co-transporter 2 (SGLT2) inhibitors (dapagliflozin, empagliflozin) to reduce adverse cardiovascular or renal outcomes (I, A).

Akpay Sarybaev

National Centre for Cardiology and Internal Medicine, Bishkek, Kyrgyz Republic

Peer-review: Internal Conflict of interest: None to declare Authorship: A.S. Acknowledgement and funding: None to declare

Address for correspondence: Akpay Sarybaev, National Centre for Cardiology and Internal Medicine, Bishkek, Kyrgyz Republic, Email: ak_sar@mail.ru

Received: 10.10.2021 Accepted: 11.10.2021

Copyright (02021 Heart, Vessels and Transplantation doi: $10.24969 /$ hvt.2020.277 


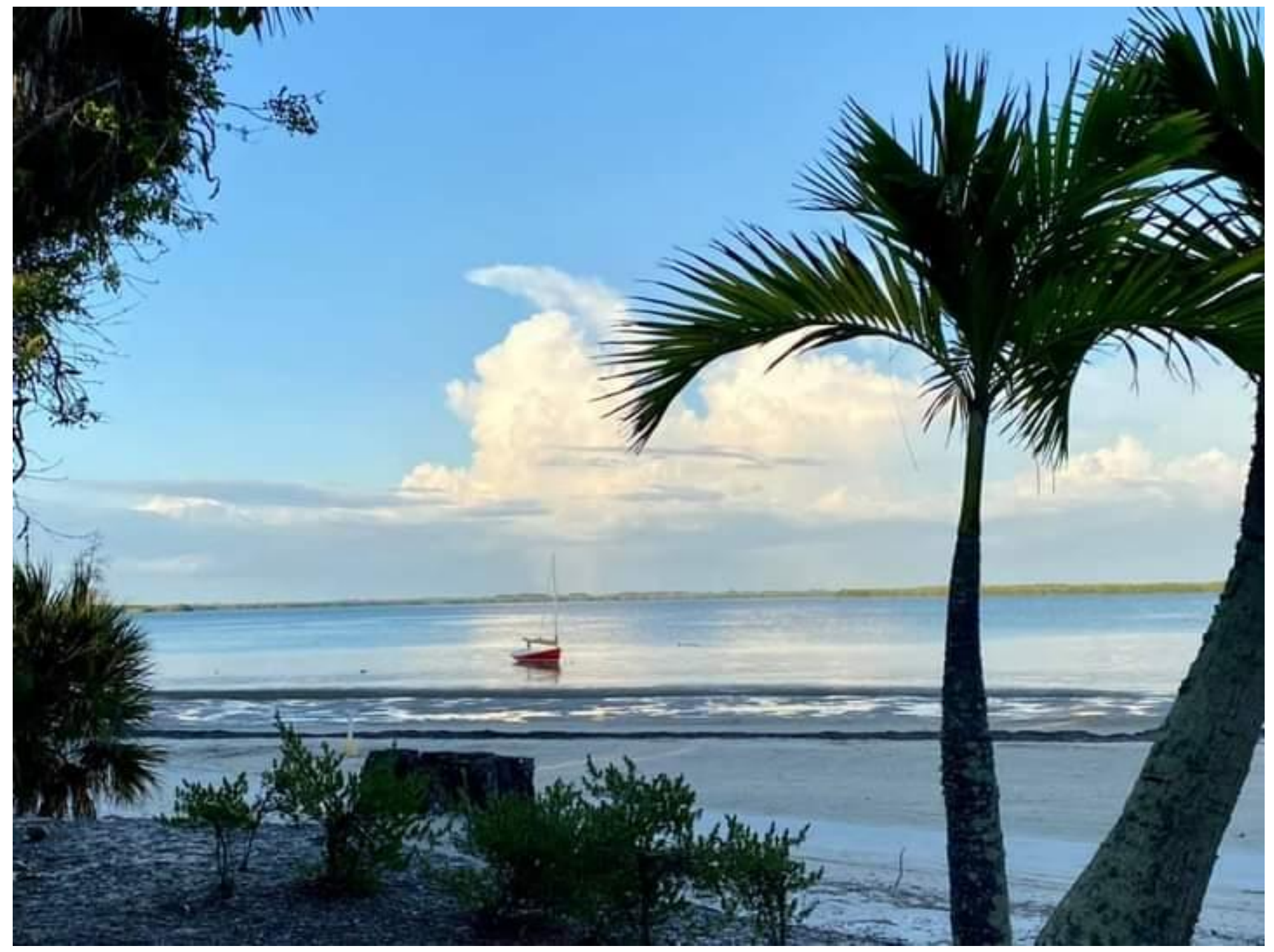

Upsula Ireland, Florida, USA. Raymond Singer, Philadelphia, PA, USA 